\title{
A Robust Estimate Method for Damage Detection of Concrete Structures Using Contaminated Data
}

\author{
X. Peng $\mathbb{D}^{1,2}$ F. J. Qin $\mathbb{D}^{1}{ }^{3}$ Q. W. Yang, ${ }^{1,2}$ and H. Chen ${ }^{4}$ \\ ${ }^{1}$ School of Civil and Transportation Engineering, Ningbo University of Technology, Ningbo 315211, China \\ ${ }^{2}$ Engineering Research Center of Industrial Construction in Civil Engineering of Zhejiang, Ningbo University of Technology, \\ Ningbo 315211, China \\ ${ }^{3}$ Key Laboratory of New Technology for Construction of Cities in Mountain Area, Ministry of Education, \\ School of Civil Engineering, Chongqing University, Chongqing 400045, China \\ ${ }^{4}$ Department of Civil Engineering, Shaoxing University, Shaoxing 312000, China
}

Correspondence should be addressed to F. J. Qin; qinfengjiang@cqu.edu.cn

Received 18 November 2020; Revised 7 December 2020; Accepted 15 April 2021; Published 12 May 2021

Academic Editor: Alessandro Palmeri

Copyright ( 12021 X. Peng et al. This is an open access article distributed under the Creative Commons Attribution License, which permits unrestricted use, distribution, and reproduction in any medium, provided the original work is properly cited.

\begin{abstract}
Damage detection of concrete structures based on finite element model and measured response parameters has been an important research topic in recent years. It is well known that test data of mechanical behavior of concrete show great scatterness. As a result, the measured response parameters of concrete structures sometimes have gross errors. The gross error is a physical quantity that is much larger than data noise, which may lead to serious distortion of calculation results. To this end, a new robust estimate method termed as the augmented inverse estimate is proposed in this work for damage detection of concrete structures to resist gross errors in data. It has the advantages of very simple programming, convenient utilization, high computational accuracy, and broad prospect of application. Central to the augmented inverse estimate are the augmentation of coefficient matrix and the multiple computations based on feedback evaluation. A reinforced concrete beam structure is used as an example to verify the proposed method. It was found that the proposed method can successfully identify the location and extent of structural damage even if the used data have gross errors.
\end{abstract}

\section{Introduction}

Damage in reinforced concrete structures accumulates gradually because of effects of cyclic load, circumstance erosion, material aging, and other deficiencies during their service life. Structural damage often leads to changes in the dynamic response parameters of the structure. By testing the vibration parameters and observing their changes, structural damage can be detected in a timely manner to avoid disastrous consequences. In recent decades, damage detection of concrete structures has become a key issue in the field of civil engineering. The method based on finite element model (FEM) updating [1-4] is one of the mainstream methods for structural damage detection, since it can simultaneously determine the locations and extents of structural damages. The basic idea of this type of method is to modify structural
FEM to match the testing parameters such as natural frequencies and mode shapes. The resulting modification of the FEM will indicate the locations and extents of structural damage. Depending on the type of parameters used, the FEM updating methods can be divided into frequency based methods [5-13], mode shape based methods [14-17], flexibility based methods [18-23], displacement based methods [24-26], and so on. In most cases, these FEM updating methods will come down to the solution of a system of linear equations. It is known that the ill-conditioned least-squares problems often arise in the computation process of solving the linear equations. This means that little errors in testing data may lead to very large errors in results of damage detection. To solve this problem, many researchers have used the biased estimate techniques for achieving a stable and accurate solution. For example, the singular-value- 
truncation (SVT) estimate [27-34] is one of the most used biased estimate techniques in last decades. The key step of the SVT estimate is to ignore the smaller singular values of the coefficient matrix of the linear equations. It has been shown that the SVT estimate has certain antinoise ability and can obtain stable calculation results.

Although much progress has been made in SVT estimate, there are still several problems that need to be addressed better. The first challenge is the gross errors that may exist in the testing data. For concrete structures, the gross errors are more likely to occur due to the great scatterness of mechanical behavior of concrete. From the point of view of absolute value, the gross error is much larger than data noise. Up to now, the existing SVT estimate is powerless for gross errors. In other words, the solution of linear equations will be seriously distorted if the testing data contain a few individual gross errors. The second problem is how to fully use the particularity of damage detection to further improve the accuracy of the calculation results. For the damage detection problem, the damaged elements in the structure are often only a small minority because the actual damage usually occurs only in a few local areas. This particularity of damage detection has not been fully utilized in the previous calculation methods.

In view of the above two problems, it is very necessary to further study the new robust estimate technique for damage detection of concrete structures using the response data with gross errors. In this paper, a new robust estimate method termed as the augmented inverse estimate (AIE) is proposed for damage detection of concrete structures. Central to the proposed method are the augmentation of coefficient matrix and the multiple computations based on feedback evaluation. The proposed AIE has three main advantages. The first advantage of AIE is the ability of resisting gross errors. The AIE can determine the quantity of gross errors and eliminate the adverse effects of gross errors. The second advantage of AIE is the conciseness in theory and very simple programming, since this method only uses the matrix generalized inverse technique. The third advantage of AIE is the high computational accuracy and broad prospect of application. The AIE has a wider application range than the SVT estimate, since AIE can resist both gross errors and noise but SVT can only resist noise. If there are no gross errors in the used data, the AIE has the same antinoise ability as the SVT estimate. If there are gross errors in the used data, the AIE performs much better than the SVT estimate, since the latter will be distorted due to gross errors. In addition, the particularity of damage detection is fully utilized in the proposed AIE by removing many undamaged elements in each computation according to the feedback evaluation. This operation can significantly reduce the computational complexity and obtain more accurate damage detection results gradually.

The remainder of this paper is organized as follows: In Section 2, the damage detection equations using frequency and mode shape sensitivities are briefly reviewed.
Subsequently, the SVT estimate for solving the damage detection equations is illustrated in Section 3. In Section 4, the proposed AIE technique is presented in detail to deal with both gross errors and noise. In Section 5, a reinforced concrete beam structure is employed as the numerical example to verify the proposed method. At last, the conclusions of this work are summarized in Section 6.

\section{Damage Detection Equations}

As stated previously, damage often leads to changes in the dynamic response parameters of the concrete structures. In this section, the damage detection equations based on vibration frequency and mode shape sensitivities are briefly reviewed. Consider a structure with $n$ degrees of freedom (DOFs), whose vibration modes can be computed by solving the following generalized eigenvalue problem:

$$
K \varphi_{j}=\lambda_{j} M \varphi_{j},
$$

where $M$ and $K$ are the mass and stiffness matrices; $\lambda_{j}$ and $\varphi_{j}$ are the $j$ th eigenvalue (squares of frequency) and eigenvector (mode shape), respectively. In general, matrix $M$ is approximately constant when damage occurs. Then the firstorder sensitivities of the jth eigenvalue and eigenvector can be computed using the formulas presented by Fox and Kapoor [35] as

$$
\begin{aligned}
& \frac{\partial \lambda_{j}}{\partial \alpha_{i}}=\varphi_{j}^{T} K_{i} \varphi_{j}, \\
& \frac{\partial \varphi_{j}}{\partial \alpha_{i}}=\sum_{r=1}^{n} \frac{\varphi_{r}^{T} K_{i} \varphi_{j}}{\lambda_{j}-\lambda_{r}} \varphi_{r}, \quad(r \neq j),
\end{aligned}
$$

where $\alpha_{i}$ and $K_{i}$ are the $i$ th elemental damage parameter and stiffness matrix, respectively. Note that the computation of the eigenvector sensitivity using equation (3) is very computationally expensive, since it requires the availability of all the mode shapes of the intact structure. In view of this, the formula presented by Yang and Peng [36] is used in this paper to compute the mode shape sensitivity as

$$
\frac{\partial \varphi_{j}}{\partial \alpha_{i}}=\left(K-\lambda_{j} M+\lambda_{j} \varphi_{j} \varphi_{j}^{T} M\right)^{-1}\left(\frac{\partial \lambda_{j}}{\partial \alpha_{i}} M-K_{i}\right) \varphi_{j} .
$$

This new formula has the advantage of requiring only the knowledge of the eigenvector to be differentiated. Thus, it is efficient and convenient in programming.

Before and after damage occurs, the changes of the $j$ th eigenvalue and eigenvector are obtained by

$$
\begin{aligned}
\Delta \lambda_{j} & =\lambda_{d j}-\lambda_{j}, \\
\Delta \varphi_{j} & =\varphi_{d j}-\varphi_{j},
\end{aligned}
$$

where $\lambda_{d j}$ and $\varphi_{d j}$ are the $j$ th eigenvalue and eigenvector of the damaged structure; $\Delta \lambda_{j}$ and $\Delta \varphi_{j}$ are the corresponding changes, respectively. Using Taylor's series expansion, the 
first-order approximations for $\Delta \lambda_{j}$ and $\Delta \varphi_{j}$ can be expressed as

$$
\begin{aligned}
\Delta \lambda_{j} & =\sum_{i=1}^{N} \alpha_{i} \frac{\partial \lambda_{j}}{\partial \alpha_{i}} \\
\Delta \varphi_{j} & =\sum_{i=1}^{N} \alpha_{i} \frac{\partial \varphi_{j}}{\partial \alpha_{i}}
\end{aligned}
$$

where $N$ is the total number of elements. For $m$ measured modes and $r$ measured DOFs, the first-order sensitivity equation can be written from equations (6) and (7) as

$$
\begin{aligned}
& \{\Delta \lambda\}=S_{1}\{\alpha\}, \\
& \{\Delta \varphi\}=S_{2}\{\alpha\},
\end{aligned}
$$

where $\quad\{\Delta \lambda\}=\left(\Delta \lambda_{1}, \Delta \lambda_{2}, \ldots, \Delta \lambda_{m}\right)^{T}, \quad\{\Delta \varphi\}=\left[\Delta \varphi_{1}^{1}, \ldots, \Delta \varphi_{1}^{r} \mid\right.$ $\left.\Delta \varphi_{2}^{1}, \ldots, \Delta \varphi_{2}^{r}|, \ldots,| \Delta \varphi_{m}^{1}, \ldots, \Delta \varphi_{m}^{r}\right]^{T}, \quad\{\alpha\}=\left(\alpha_{1}, \alpha_{2}, \ldots, \alpha_{N}\right)^{T}$, and $S_{1}$ and $S_{2}$ are the corresponding sensitivity matrices, respectively. Combining equation (8) with (9), the damage detection equation can be obtained as

$$
\left[\begin{array}{l}
\{\Delta \lambda\} \\
\{\Delta \varphi\}
\end{array}\right]=\left[\begin{array}{c}
S_{1} \\
S_{2}
\end{array}\right]\{\alpha\} .
$$

Let

$$
\begin{aligned}
{\left[\begin{array}{l}
\{\Delta \lambda\} \\
\{\Delta \varphi\}
\end{array}\right] } & =b, \\
{\left[\begin{array}{c}
S_{1} \\
S_{2}
\end{array}\right] } & =A, \\
\{\alpha\} & =x .
\end{aligned}
$$

Then, equation (10) can be simplified as

$$
A \cdot x=b \text {. }
$$

Finally, the elemental damage coefficients $x$ can be obtained by solving the linear equation (12). According to the resulting $x$, the locations and extents of damage in the concrete structure can be evaluated.

\section{SVT Estimate}

Next, we discuss the specific methods to solve the linear equation (12). The generalized inverse technique [37-40] is used in many cases to compute $x$ in equation (12); that is,

$$
x=A^{+} b,
$$

where the superscript "+" denotes the Moore-Penrose generalized inverse. According to the matrix theory, the generalized inverse of a matrix can be computed by singular value decomposition (SVD) technique. The SVD of matrix $A$ can be expressed as

$$
\begin{aligned}
& A=U \Lambda V^{T}, \\
& U=\left[u_{1}, u_{2}, \ldots, u_{n}\right], \\
& V=\left[v_{1}, v_{2}, \ldots, v_{N}\right], \\
& \Lambda=\left[\begin{array}{ll}
Z & 0 \\
0 & 0
\end{array}\right], \\
& Z=\operatorname{diag}\left(\sigma_{1}, \sigma_{2}, \ldots, \sigma_{t}\right),
\end{aligned}
$$

where $U$ and $V$ are the orthogonal matrices, $\sigma_{1}, \sigma_{2}, \ldots, \sigma_{t}$ are the nonzero singular values of $A$ with $\sigma_{1} \geq \sigma_{2} \geq \cdots \geq \sigma_{t}$. From equations (14)-(17), the generalized inverse of $A$ can be computed by

$$
A^{+}=\sum_{x=1}^{t} \sigma_{x}^{-1} v_{x} u_{x}^{T} .
$$

Substituting equation (18) into (13), one has

$$
x=\left(\sum_{x=1}^{t} \sigma_{x}^{-1} v_{x} u_{x}^{T}\right) \cdot b .
$$

From equation (19), one can see that small singular values have greater influence on the accuracy of the solution. Thus, it is a feasible way to improve the stability of the solution by ignoring the small singular values in equation (19). This is the core idea of the SVT estimate method. Then the solution of the SVT estimate is expressed by ignoring some smaller singular values as

$$
x_{\mathrm{svt}}=\left(\sum_{x=1}^{z} \sigma_{x}^{-1} v_{x} u_{x}^{T}\right) \cdot b \text {, }
$$

where $z$ is the number of remaining singular values. The main problem existing in SVT estimate is how to choose a suitable value of the number of remaining singular values. The $L$-curve method [41-43] will be used in this paper for choosing the number of remaining singular values. It is known that SVT estimate has the ability of resisting data noise but not the ability of resisting gross errors.

\section{The Proposed AIE Method}

As stated previously, there may be gross errors in the testing data due to the great scatterness of mechanical behavior of concrete. The existence of gross errors will lead to serious distortion of the results obtained by SVT estimate. In view of this, a new robust estimate method termed as the augmented inverse estimate is proposed in this section for resisting both gross errors and noise. The main innovations of the AIE method lie in the augmentation of coefficient matrix and multiple computations based on feedback evaluation. The AIE method is illustrated in detail as follows. 
Firstly, each element in the observation vector $b$ is successively assumed to be the data with gross error. Without loss of generality, assuming that the element containing the gross error is $b_{i}$, the linear equation (12) can be expressed as

$$
A \cdot x=\left\{\begin{array}{c}
b_{1} \\
\vdots \\
b_{i} \\
\vdots \\
b_{m(r+1)}
\end{array}\right\} .
$$

Element $b_{i}$ in equation (21) cannot be used if it contains the unknown gross error. Assuming that the true value is $\delta_{i}$, equation (21) should be rewritten as

$$
A \times x=\left\{\begin{array}{c}
b_{1} \\
\vdots \\
\delta_{i} \\
\vdots \\
b_{m(r+1)}
\end{array}\right\} .
$$

The unknowns $x$ and $\delta_{i}$ can be synthesized into a new unknown vector $\bar{x}$ by rewriting equation (22) as

$$
\bar{A} \cdot \bar{x}=\bar{b},
$$

$$
\begin{gathered}
\bar{A}=\left[\begin{array}{c}
0 \\
\vdots \\
A \mid-1 \\
\vdots \\
0
\end{array}\right], \\
\bar{x}=\left[\begin{array}{c}
x_{1} \\
\vdots \\
x_{N} \\
\delta_{i}
\end{array}\right], \\
\bar{b}=\left[\begin{array}{c}
b_{1} \\
\vdots \\
0 \\
\vdots \\
b_{m(r+1)}
\end{array}\right],
\end{gathered}
$$

where $\bar{A}$ is the augmented coefficient matrix and $\bar{x}$ is the augmented unknown vector. Then the new linear equation (23) can be solved again by the SVT as described from equation (14) to equation (20). That is,

$$
\begin{aligned}
\bar{x} & =\left(\sum_{x=1}^{z} \bar{\sigma}_{x}^{-1} \bar{v}_{x} \bar{u}_{x}^{T}\right) \cdot \bar{b}, \\
x_{\mathrm{AIE}} & =\{\bar{x}\}_{N}^{1},
\end{aligned}
$$

$$
\delta_{i}=\{\bar{x}\}_{N+1} .
$$

Equations (28) and (29) are the calculation formulas of AIE solution, where $x_{\text {AIE }}$ denotes the AIE solution of the damage parameters, and $\{\bar{x}\}_{N}^{1}$ denotes the first $N$ coefficients of $\bar{x}$ obtained by equation (27). According to $\{\bar{x}\}_{N}^{1}$, the possible damage locations and extents can be assessed. Equation (29) can be used to calculate the value of the gross error by $b_{i}-\delta_{i}$, if it is necessary. Note that the above derivation process can be easily extended to the case of more than one gross error. Without loss of generality, the relevant computation formulas for the case of two gross errors are presented in the following. Assuming that the elements containing the gross errors are $b_{i}$ and $b_{j}$, the linear equation (12) can be revised by replacing $b_{i}\left(b_{j}\right)$ with $\delta_{i}\left(\delta_{j}\right)$ as

$$
A \times x=\left\{\begin{array}{c}
b_{1} \\
\vdots \\
\delta_{i} \\
\vdots \\
\delta_{j} \\
\vdots \\
b_{m(r+1)}
\end{array}\right\} .
$$

As before, the unknowns $x, \delta_{i}$, and $\delta_{j}$ can be synthesized into a new unknown vector $\overline{\bar{x}}$ by rewriting equation (30) as

$$
\begin{aligned}
& \overline{\bar{A}} \cdot \overline{\bar{x}}=\overline{\bar{b}},
\end{aligned}
$$

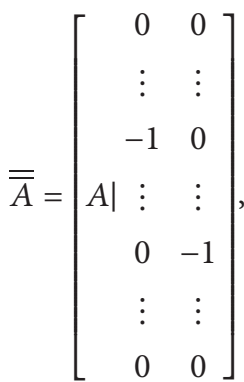

$$
\begin{aligned}
& \overline{\bar{x}}=\left[\begin{array}{c}
x_{1} \\
\vdots \\
x_{N} \\
\delta_{i} \\
\delta_{j}
\end{array}\right] \text {, } \\
& \overline{\bar{b}}=\left\{\begin{array}{c}
b_{1} \\
\vdots \\
0 \\
\vdots \\
0 \\
\vdots \\
b_{m(r+1)}
\end{array}\right\} \text {, }
\end{aligned}
$$


where $\overline{\bar{A}}$ is the new augmented coefficient matrix, and $\overline{\bar{x}}$ is the new augmented unknown vector. Then the new solution is

$$
\begin{aligned}
\overline{\bar{x}} & =\left(\sum_{x=1}^{z} \overline{\bar{\sigma}}_{x}^{-1} \overline{\bar{v}}_{x} \overline{\bar{u}}_{x}^{T}\right) \cdot \overline{\bar{b}}, \\
x_{\mathrm{AIE}} & =\{\overline{\bar{x}}\}_{N}^{1}, \\
\delta_{i} & =\{\overline{\bar{x}}\}_{N+1}, \\
\delta_{j} & =\{\overline{\bar{x}}\}_{N+2} .
\end{aligned}
$$

Again, equation (33) is the calculation formula of AIE solution for the damage parameters. Equations (34) and (35) can be used to calculate the values of the gross errors by $b_{i}-\delta_{i}$ and $b_{j}-\delta_{j}$, if it is necessary.

Finally, we discuss the multiple computations process based on feedback evaluation for the damage detection problem. In general, the perturbed elements in the FEM due to structural damages are often only a small minority. This particularity will result in the existence of a large number of coefficients close to zero in $x_{\mathrm{AIE}}$ obtained by equation (28). Thus, these coefficients close to zero in $x_{\mathrm{AIE}}$ should be seen as a product of data noise and they can be set to zeros to simplify equation (22) for the next recalculation. Generally, those values in $x_{\mathrm{AIE}}$ which satisfy $\left(x_{i} / \max \left(x_{\mathrm{AIE}}\right)\right) \leq 0.05$ should be deemed to correspond to those undamaged elements in the structure. Then equation (23) can be further simplified for the recalculation by removing some column vectors in $\bar{A}$ and coefficients in $\bar{x}$ corresponding to those undamaged elements; that is,

$$
\bar{A}^{\prime} \cdot \bar{x}^{\prime}=\bar{b},
$$

where $\bar{A}^{\prime}$ is the remaining matrix of $\bar{A}$ after removing some column vectors related to those undamaged elements and $\bar{x}^{\prime}$ is the remaining vector of $\bar{x}$ after removing the corresponding coefficients. From equation (36), the second AIE solution can be obtained again using the SVT as

$$
\bar{x}^{\prime}=\left(\sum_{x=1}^{z} \bar{\sigma}_{x}^{\prime}-1 \bar{v}_{x}^{\prime} \bar{u}_{x}^{T}\right) \cdot \bar{b} .
$$

The above recalculation process like equations (36) and (37) should be repeated until there are no coefficients in $x_{\text {AIE }}^{v}$ which satisfy $\left(x_{i} / \max \left(x_{\mathrm{AIE}}^{v}\right)\right) \leq 0.05$, where the superscript $v$ denotes the $v$ th AIE computation. In the end, the true damaged elements can be identified according to the final AIE solution. In the above process, it is important to note that the computational complexity of each AIE computation gradually decreases, since the number of unknowns decreases gradually.

In the end, the operation steps of this method are summarized as follows:

(1) Establish the FEM of the structure and compute the eigenvalue and eigenvector sensitivities using equations (2) and (4).
(2) Measure the modal parameters of the damaged structure and establish the damage detection basic equation (12).

(3) Use AIE method to calculate the damage parameters and possible gross errors. Repeat this operation multiple times by feedback evaluation until the true damaged elements can be identified according to the final AIE solution.

\section{Numerical Validation and Comparative Study}

The reinforced concrete beam shown in Figure 1 is used to verify the proposed method for damage detection. The cross section of the structure is rectangular of $110 \mathrm{~mm} \times 180 \mathrm{~mm}$. The structure is divided into 32 beam elements and the length of each element is $70 \mathrm{~mm}$. Each node has three degrees of freedom, that is, two translational degrees of freedom and one rotational degree of freedom. The physical parameters of this structure are as follows: elasticity moduli of concrete and steel are $E_{c}=32.5 \mathrm{GPa}$ and $E_{s}=200 \mathrm{GPa}$; Poisson's ratio $v=0.2$; and density $\rho=2650 \mathrm{~kg} / \mathrm{m}^{3}$. Two damage cases are simulated: (1) element 15 has $20 \%$ stiffness reduction; (2) elements 6 and 18 both have 15\% stiffness reductions. In the following computation, only the firstorder vibration modal parameters are used to simulate the incomplete measurement in engineering practice. Moreover, only the data corresponding to vertical degrees of freedom of the mode shape are used in the computation because of the difficulty in measuring the rotational degrees of freedom. In addition, $2 \%$ noise is added to the simulated fundamental frequency mode to simulate the measurement error and the data at the 9th measuring point is magnified 10 times to simulate the gross error. Figure 2 presents the damage detection result using the SVT for the simulated damage case 1. One can see from Figure 2 that the SVT solution is seriously distorted, since element 15 cannot be determined to be the most possible damaged element. This means that the SVT method cannot effectively overcome the adverse effect of the gross error. Using the proposed AIE method, Figures 3-5 give the first, second, and third calculation results. One can see from these figures that more accurate detection results can be obtained gradually using the AIE method. From Figure 5, the satisfactory result is achieved after third AIE computations with feedback evaluations. This final assessment as shown in Figure 5 can clearly indicate that only element 15 is damaged and the calculated value of the damage parameter is $\alpha_{15}=0.246$. It has been shown that the proposed AIE method can effectively overcome the adverse effects of the gross error and data noise. For the second damage case, Figures 6-10 provide the calculation results obtained by the SVT, the first to fourth AIE, respectively. Apparently, the SVT solution shown in Figure 6 cannot indicate that elements 6 and 18 are damaged. The result again demonstrates that the SVT is powerless to resist the gross error. Using the proposed AIE method, satisfactory identification results as shown in Figure 10 can be obtained after four feedback calculations. This again demonstrates the 


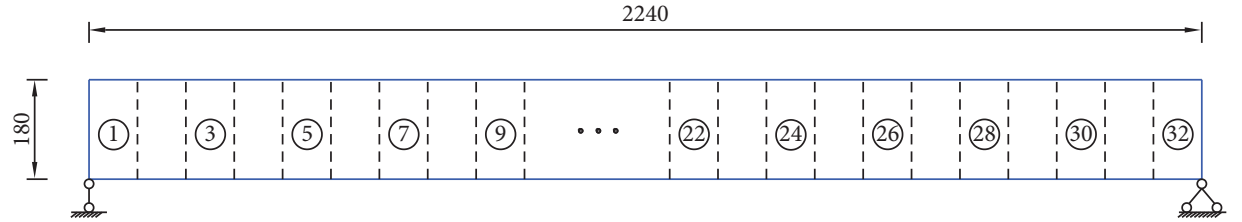

FIgURE 1: A reinforced concrete beam (unit: $\mathrm{mm}$ ).

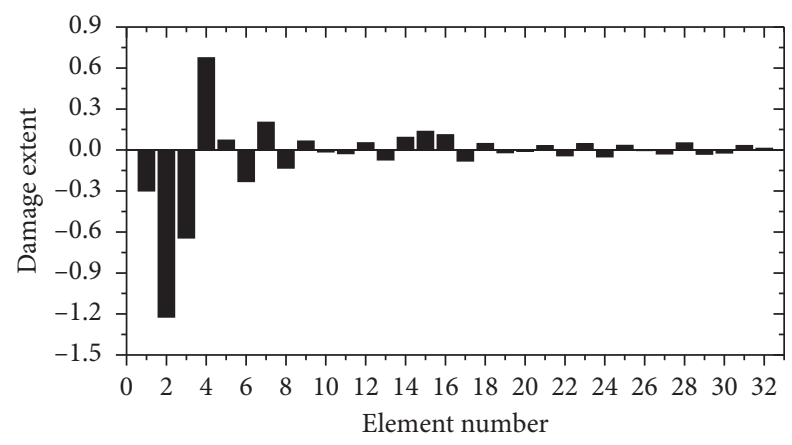

SVT solution

FIgURE 2: Damage detection result by SVT solution when element 15 is damaged.

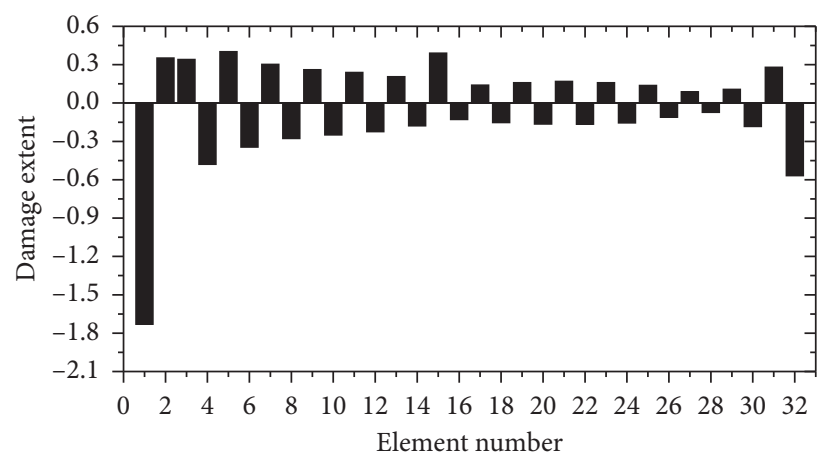

The first AIE solution

FIGURE 3: Damage assessment result by the first AIE when element 15 is damaged.

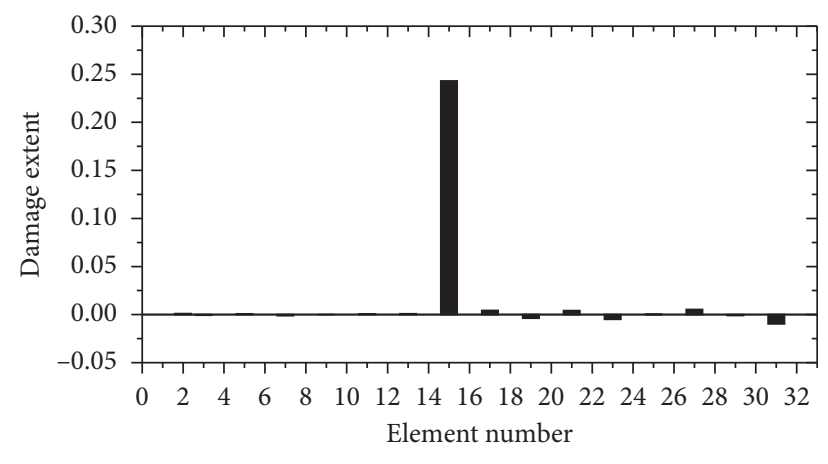

The second AIE solution

FIgURE 4: Damage assessment result by the second AIE when element 15 is damaged. 


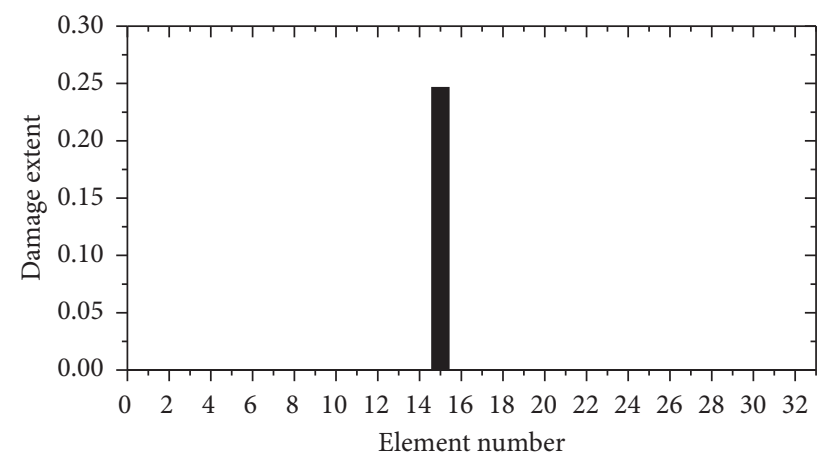

The third AIE solution

FIgURE 5: Damage assessment result by the third AIE when element 15 is damaged.

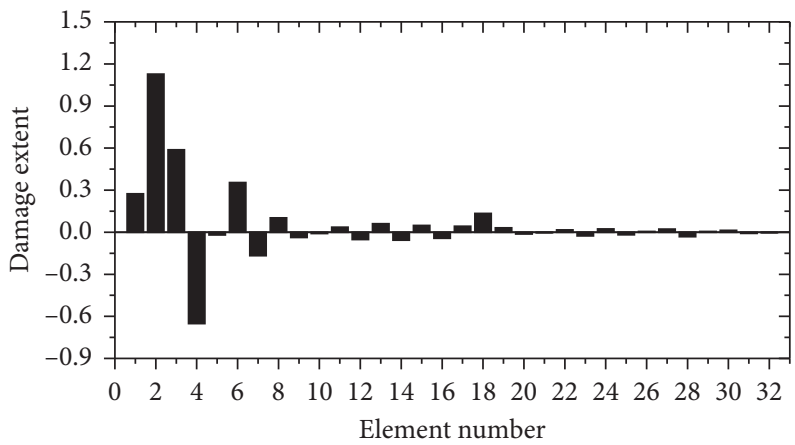

SVT solution

FIGURE 6: Damage assessment result by the SVT when elements 6 and 18 are damaged.

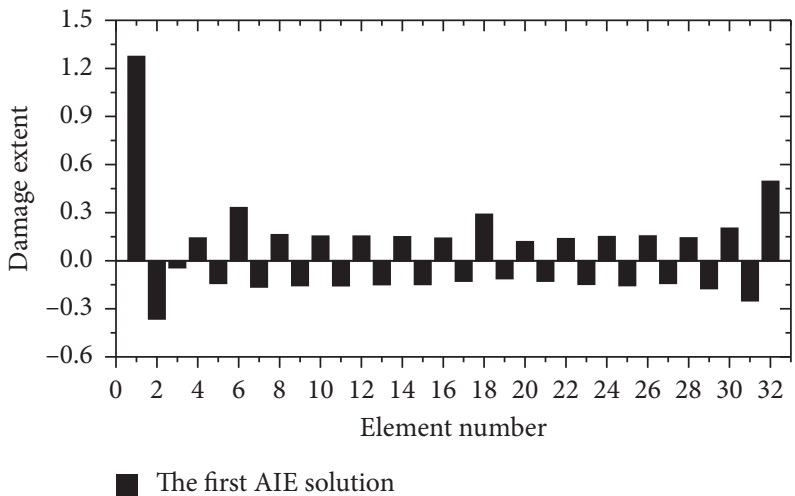

Figure 7: Damage assessment result by the first AIE when elements 6 and 18 are damaged.

superiority and robustness of the proposed AIE method in solving the damage detection problem under gross error pollution.

Next, the case of two gross errors is simulated to further verify the proposed method. The data at the 9th and 20th measuring points are magnified 10 and 5 times to simulate the gross errors, respectively. Figure 11 presents the damage detection result by SVT solution. It is clear that the result is not accurate due to the fact that SVT cannot overcome the adverse effects of gross errors. Using the proposed method, Figures 12-15 give the damage detection results by the first to fourth AIE solutions. Apparently, the true damaged element 21 can be identified according to Figure 15. As a byproduct, the calculated data at the 9th and 20th measuring points can be simultaneously obtained. The deviation ratios of the 9th and 20th data are 8.6931 and 3.1842. This means 


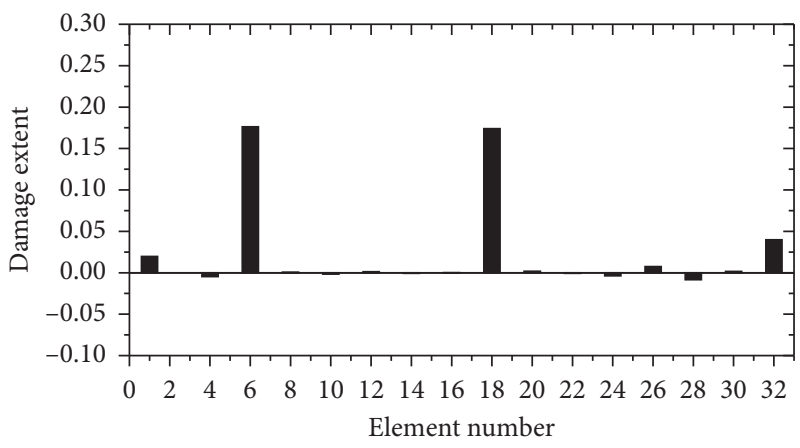

The second AIE solution

Figure 8: Damage assessment result by the second AIE when elements 6 and 18 are damaged.

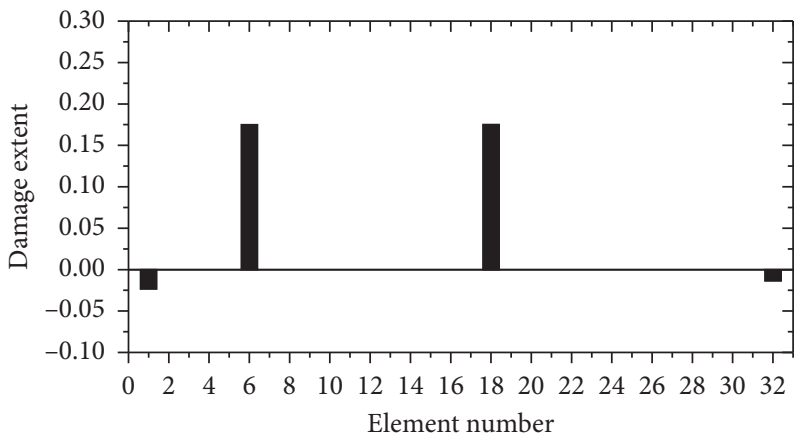

The third AIE solution

FIgURE 9: Damage assessment result by the third AIE when elements 6 and 18 are damaged.

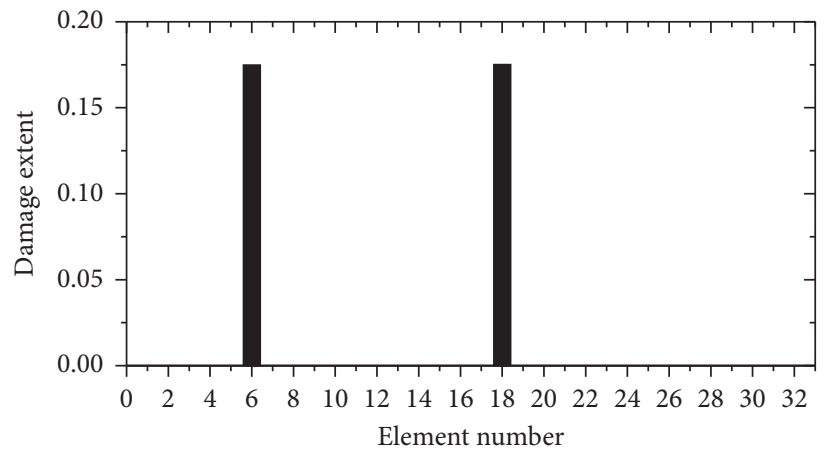

The fourth AIE solution

Figure 10: Damage assessment result by the fourth AIE when elements 6 and 18 are damaged.

that the 9th and 20th data obviously have gross errors. This result is consistent with the hypothesis which shows that the proposed method is reasonable and feasible.

In order to compare the advantages and disadvantages of the existing singular-value-truncation algorithm (SVT) and the augmented inverse estimate (AIE), $\sigma_{\text {damage }}$ is used to describe the error degrees of damage assessment:

$$
\sigma_{\text {damage }}=\sqrt{\frac{\sum_{i=1}^{n}\left(x-x_{0}\right)^{2}}{n}} .
$$

The error degrees of damage assessment under different methods calculated by equation (38) are shown in Table 1.

From Table 1, $\sigma_{\text {damage }}$ calculated by SVT method has a high degree of dispersion, with a maximum value of $83.9 \%$, which occurs when element 21 is damaged. With the increase of the number of iterations, $\sigma_{\text {damage }}$ using AIE method decreases obviously. In all cases, $\sigma_{\text {damage }}$ is less than $1 \%$ by the fourth AIE. It can be seen that the AIE method has greatly improved the accuracy of the evaluation. 


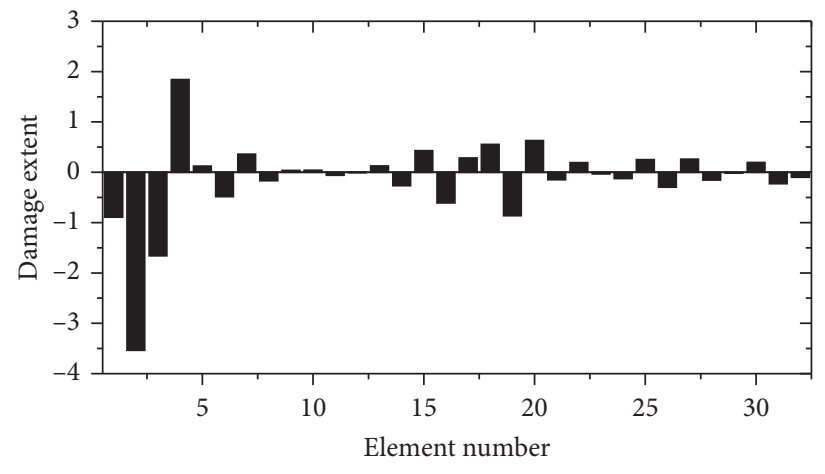

SVT solution

FIGURE 11: Damage detection result by SVT solution when element 21 is damaged.

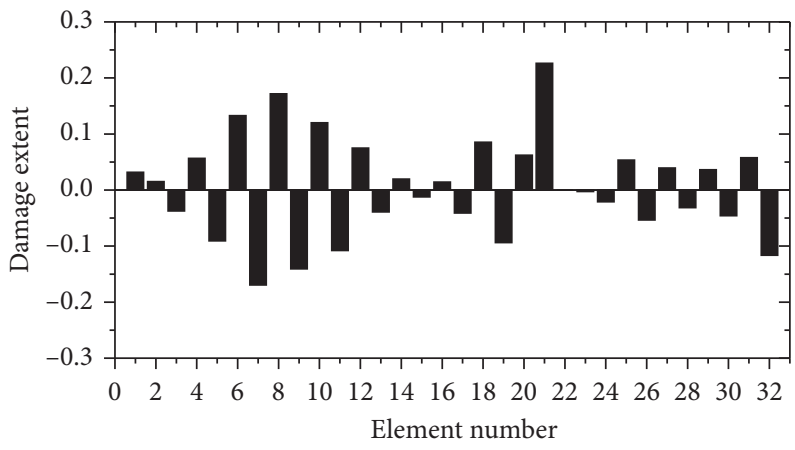

The first AIE solution

Figure 12: Damage assessment result by the first AIE when element 21 is damaged.

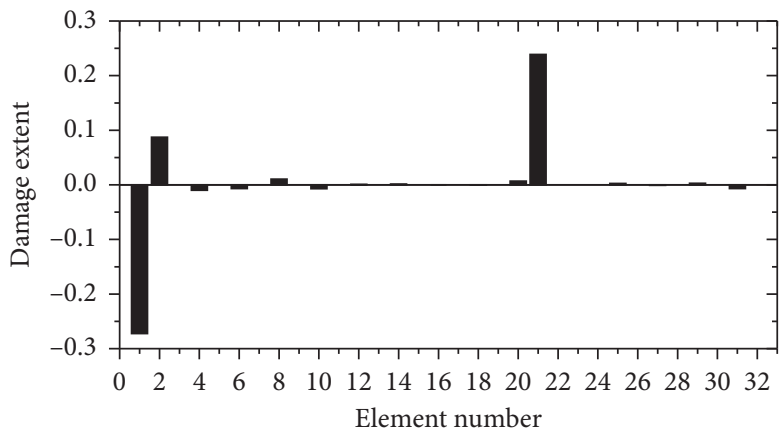

The second AIE solution

FIGURE 13: Damage assessment result by the second AIE when element 21 is damaged. 


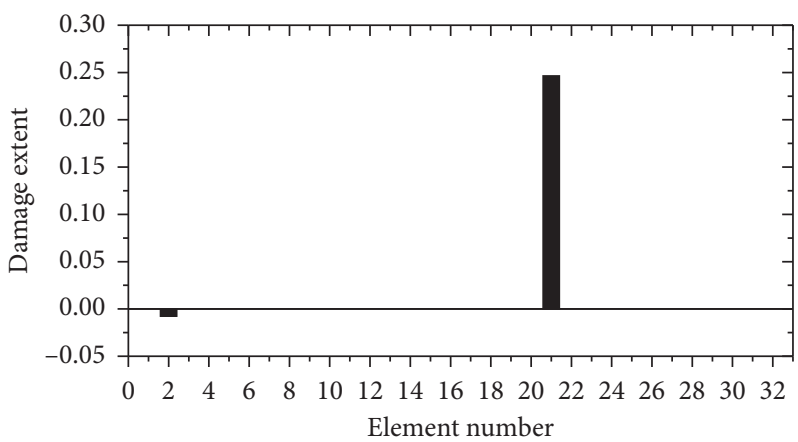

The third AIE solution

Figure 14: Damage assessment result by the third AIE when element 21 is damaged.

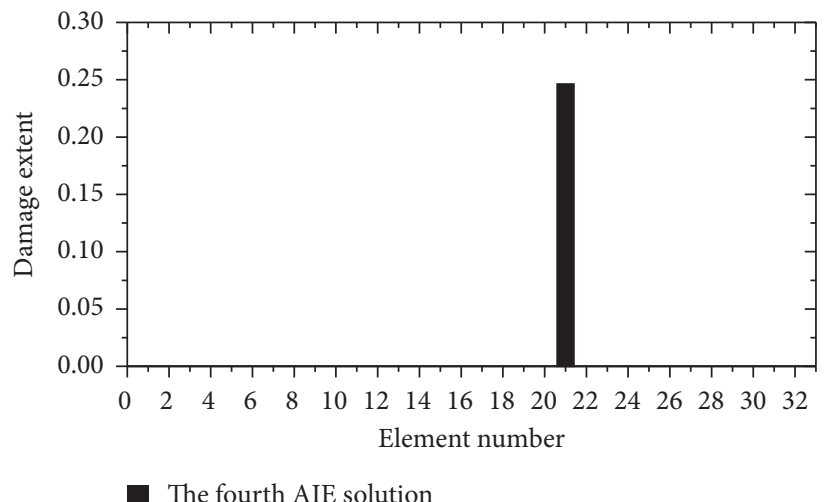

FIGURe 15: Damage assessment result by the fourth AIE when element 21 is damaged.

TABLE 1: The error degrees of damage assessment by different methods.

\begin{tabular}{lcccccc}
\hline Case & Location of damage & SVT (\%) & First AIE (\%) & Second AIE (\%) & Third AIE (\%) & Fourth AIE (\%) \\
\hline 1 & Element 15 & 28.6 & 39.3 & 0.8 & 0.8 & 0.8 \\
2 & Elements 6 and 18 & 26.3 & 28.9 & 1.0 & 0.8 & 0.6 \\
3 & Element 21 & 83.9 & 7.8 & 5.1 & 0.8 & 0.8 \\
\hline
\end{tabular}

\section{Conclusion}

In this paper, a new robust estimate method termed as the augmented inverse estimate (AIE) is proposed for damage detection of concrete structures to resist gross errors in data. The proposed method has the advantages of very simple programming, convenient utilization, high computational accuracy, and broad prospect of application. Central to the augmented inverse estimate (AIE) method are the augmentation of coefficient matrix and the multiple computations based on feedback evaluation. A reinforced concrete beam structure is used to verify the proposed augmented inverse estimate method. It was found that the augmented inverse estimate (AIE) method can achieve more accurate damage detection results compared with the existing singular-value-truncation (SVT) algorithm. It may be a promising tool for solving the damage detection problem of concrete structures when the used data have gross errors.
Further research on the technique can be carried out to deal with damage detection with fewer data, to compare the technique with other methods, and to verify the technique by experiment.

\section{Data Availability}

The data used to support the findings of this study are available from the corresponding author upon request.

\section{Conflicts of Interest}

The authors declare that they have no conflicts of interest.

\section{Acknowledgments}

This work was supported by the National Natural Science Foundation of China (52008215); the Natural Science 
Foundation of Zhejiang Province, China (LQ20E080013); and the major special science and technology project (2019B10076) of "Ningbo Science and Technology Innovation 2025."

\section{References}

[1] P. B. Rubio, F. Louf, and L. Chamoin, "Fast model updating coupling Bayesian inference and PGD model reduction," Computational Mechanics, vol. 62, pp. 1-25, 2018.

[2] A. C. Altunişik, O. Ş Karahasan, A. F. Genç et al., "Sensitivitybased model updating of building frames using modal test data," KSCE Journal of Civil Engineering, vol. 22, pp. 1-9, 2018.

[3] D. T. Bartilson, J. Jang, and A. W. Smyth, "Finite element model updating using objective-consistent sensitivity-based parameter clustering and Bayesian regularization," Mechanical Systems and Signal Processing, vol. 114, pp. 328-345, 2019.

[4] Z. Yuan, P. Liang, T. Silva, K. Yu, and J. E. Mottershead, "Parameter selection for model updating with global sensitivity analysis," Mechanical Systems and Signal Processing, vol. 115, pp. 483-496, 2019.

[5] A. Messina, J. E. Williams, and T. Contursi, "Structural damage detection by a sensitivity and statistical-based method," Journal of Sound and Vibration, vol. 216, no. 5, p. 791 808, 1996.

[6] L. Yu, L. Cheng, L. H. Yam, and Y. J. Yan, "Application of eigenvalue perturbation theory for detecting small structural damage using dynamic responses," Composite Structures, vol. 78, no. 3, pp. 402-409, 2007.

[7] Q. W. Yang and J. K. Liu, "Structural damage assessment by adding given masses," Engineering Mechanics, vol. 26, no. 5, pp. 159-163, 2009, in Chinese.

[8] N. T. Khiem and L. K. Toan, "A novel method for crack detection in beam-like structures by measurements of natural frequencies," Journal of Sound and Vibration, vol. 333, no. 18, pp. 4084-4103, 2014.

[9] Z. Ding, Z. Lu, M. Huang, and J. Liu, "Improved artificial bee colony algorithm for crack identification in beam using natural frequencies only," Inverse Problems in Science and Engineering, vol. 25, no. 2, pp. 218-238, 2017.

[10] C. G. Krishnanunni, R. S. Raj, D. Nandan et al., "Sensitivitybased damage detection algorithm for structures using vibration data," Journal of Civil Structural Health Monitoring, vol. 9, pp. 1-15, 2018.

[11] A. J. Choi and J.-H. Han, "Frequency-based damage detection in cantilever beam using vision-based monitoring system with motion magnification technique," Journal of Intelligent Material Systems and Structures, vol. 29, no. 20, pp. 3923-3936, 2018.

[12] J. Pan, Z. Zhang, J. Wu, K. R. Ramakrishnan, and H. K. Singh, "A novel method of vibration modes selection for improving accuracy of frequency-based damage detection," Composites Part B: Engineering, vol. 159, pp. 437-446, 2019.

[13] G. D. Ercolani, D. H. Felix, and N. F. Ortega, "Crack detection in prestressed concrete structures by measuring their natural frequencies," Journal of Civil Structural Health Monitoring, vol. 8, no. 4, pp. 661-671, 2018.

[14] J.-T. Kim, Y.-S. Ryu, H.-M. Cho, and N. Stubbs, "Damage identification in beam-type structures: frequency-based method vs mode-shape-based method," Engineering Structures, vol. 25, no. 1, pp. 57-67, 2003.

[15] N. M. M. Maia, J. M. M. Silva, E. A. M. Almas, and R. P. C. Sampaio, "Damage detection in structures: from mode shape to frequency response function methods," Mechanical
Systems and Signal Processing, vol. 17, no. 3, pp. 489-498, 2003.

[16] E. Parloo, P. Guillaume, and M. Van Overmeire, "Damage assessment using mode shape sensitivities," Mechanical Systems and Signal Processing, vol. 17, no. 3, pp. 499-518, 2003.

[17] P. Qiao, K. Lu, W. Lestari, and J. Wang, "Curvature mode shape-based damage detection in composite laminated plates," Composite Structures, vol. 80, no. 3, pp. 409-428, 2007.

[18] B. Jaishi and W. X. Ren, "Damage detection by finite element model updating using modal flexibility residual," Journal of Sound and Vibration, vol. 290, no. 1-2, pp. 369-387, 2006.

[19] D. Bernal, "Flexibility-based damage localization from stochastic realization results," Journal of Engineering Mechanics, vol. 132, no. 6, pp. 651-658, 2006.

[20] E. Reynders and G. De Roeck, "A local flexibility method for vibration-based damage localization and quantification," Journal of Sound and Vibration, vol. 329, no. 12, pp. 23672383, 2010.

[21] Q. W. Yang and J. K. Liu, "Damage identification by the eigenparameter decomposition of structural flexibility change," International Journal for Numerical Methods in Engineering, vol. 78, no. 4, pp. 444-459, 2009.

[22] Q. Yang, "A new damage identification method based on structural flexibility disassembly," Journal of Vibration and Control, vol. 17, no. 7, pp. 1000-1008, 2011.

[23] Q. W. Yang, B. X. Sun, and C. Lu, "An improved spectral decomposition flexibility perturbation method for finite element model updating," Advances in Mechanical Engineering, vol. 10, no. 12, 2018.

[24] S. Caddemi and A. Morassi, "Crack detection in elastic beams by static measurements," International Journal of Solids and Structures, vol. 44, no. 16, pp. 5301-5315, 2007.

[25] Q. Yang and B. Sun, "Structural damage localization and quantification using static test data," Structural Health Monitoring, vol. 10, no. 4, pp. 381-389, 2011.

[26] N. T. Le, D. P. Thambiratnam, A. Nguyen, and T. H. T. Chan, "A new method for locating and quantifying damage in beams from static deflection changes," Engineering Structures, vol. 180, pp. 779-792, 2019.

[27] A. Neumaier, "Solving ill-conditioned and singular linear systems: a tutorial on regularization," SIAM Review, vol. 40, no. 3, pp. 636-666, 1998.

[28] M. Basseville, L. Mevel, and M. Goursat, "Statistical modelbased damage detection and localization: subspace-based residuals and damage-to-noise sensitivity ratios," Journal of Sound and Vibration, vol. 275, no. 3-5, pp. 769-794, 2004.

[29] H.-P. Chen, "Application of regularization methods to damage detection in large scale plane frame structures using incomplete noisy modal data," Engineering Structures, vol. 30, no. 11, pp. 3219-3227, 2008.

[30] B. Weber, P. Paultre, and J. Proulx, "Consistent regularization of nonlinear model updating for damage identification," Mechanical Systems and Signal Processing, vol. 23, no. 6, pp. 1965-1985, 2009.

[31] X. Y. Li and S. S. Law, "Adaptive Tikhonov regularization for damage detection based on nonlinear model updating," Mechanical Systems and Signal Processing, vol. 24, no. 6, pp. 1646-1664, 2010.

[32] Q. Huang, P. Gardoni, and S. Hurlebaus, "A probabilistic damage detection approach using vibration-based nondestructive testing," Structural Safety, vol. 38, pp. 11-21, 2012.

[33] Z. Zhao, R. Lin, Z. Meng et al., "A modified truncation singular value decomposition method for solving ill-posed 
problems," Journal of Algorithms \& Computational Technology, vol. 13, pp. 1-7, 2018.

[34] R. Arcucci, L. Mottet, C. Pain, and Y.-K. Guo, "Optimal reduced space for variational data assimilation," Journal of Computational Physics, vol. 379, pp. 51-69, 2019.

[35] R. L. Fox and M. P. Kapoor, "Rates of change of eigenvalues and eigenvectors," AIAA Journal, vol. 6, no. 12, pp. 2426-2429, 1968.

[36] Q. W. Yang and X. Peng, "An exact method for calculating the eigenvector sensitivities," Applied Sciences, vol. 4, no. 10, p. 2577, 2020.

[37] A. Ben-Israel, "An iterative method for computing the generalized inverse of an arbitrary matrix," Mathematics of Computation, vol. 19, no. 91, p. 452, 1965.

[38] D. W. Marquaridt, "Generalized inverses, ridge regression, biased linear estimation, and nonlinear estimation," Technometrics, vol. 12, no. 3, pp. 591-612, 1970.

[39] V. Pan and R. Schreiber, "An improved Newton iteration for the generalized inverse of a matrix, with applications," SIAM Journal on Scientific and Statistical Computing, vol. 12, no. 5, pp. 1109-1130, 1991.

[40] M. A. Rakha, "On the Moore-Penrose generalized inverse matrix," Applied Mathematics and Computation, vol. 158, no. 1, pp. 185-200, 2004.

[41] P. C. Hansen, "Analysis of discrete ill-posed problems by means of the L-curve," SIAM Review, vol. 34, no. 4, pp. 561-580, 1992.

[42] P. C. Hansen and D. P. O'Leary, "The use of the L-curve in the regularization of discrete ill-posed problems," SIAM Journal on Scientific Computing, vol. 14, no. 6, pp. 1487-1503, 1993.

[43] L. Reichel and H. Sadok, "A new L-curve for ill-posed problems," Journal of Computational and Applied Mathematics, vol. 219, no. 2, pp. 493-508, 2008. 